\title{
Physical Conditions and Abundances in NGC 6543
}

\author{
R. Wesson, X.-W. Liu \\ Department of Physics and Astronomy, University College London, \\ Gower Street, London WC1E 6BT
}

\section{Introduction}

We present analysis of the physical conditions and chemical abundances in NGC 6453, the Cat's Eye Nebula. Using archival HST images in conjunction with ground-based long-slit scanning spectra, we have produced an [O III] forbidden line temperature map of the nebula, which shows that the temperature remains constant at around $8000 \mathrm{~K}$ across the nebula. From the long-slit spectra, we have determined abundances both from collisionally excited lines (CELs) and optical recombination lines (ORLs). The ORL abundances are higher than the CEL abundances by a factor of approximately two.

\section{Analysis}

An extinction map of NGC 6543 was produced from the F487N $(\mathrm{H} \beta)$ and $\mathrm{F} 656 \mathrm{~N}$ $(\mathrm{H} \alpha)$ images, by comparing the observed ratio in each pixel to the theoretical value. The reddening is low, with an average logarithmic extinction at $\mathrm{H} \beta$, $c=0.1$, and exhibits only small spatial variations. Consequently, all images were dereddened using a constant $c$ value of 0.10 .

Using images taken in $H S T$ filters $\mathrm{F} 437 \mathrm{~N}$ ([O III $] \lambda 4363$ ) and F502N ([O III $]$ $\lambda 5007)$, a temperature map was produced. The [O III] $\lambda 4363$ is weak, and the filter bandpass contains significant nebular continuum emission as well as contributions from $\mathrm{H} \gamma \lambda 4340$ and $\mathrm{He}$ I $\lambda 4388$, which must be corrected for. For this purpose, we have made use of the theoretical continua from Brown \& Matthews (1970), He I recombination coefficients from Benjamin, Skillman \& Smits (1999) and $\mathrm{H}$ I recombination coefficients from Storey \& Hummer (1995) together with filter profiles from the IRAF task bandpar. The predicted nebular continuum level is further constrained using our long-slit spectrum. The correction was calculated in terms of the flux of $\mathrm{H} \beta$ only, as the ratio of images F487N \& F588N is virtually constant across the nebula. The correction applied was, $I([\mathrm{O} \mathrm{III}]$ $\lambda 4363)=\mathrm{F} 437 \mathrm{~N}-0.032 \times \mathrm{F} 487 \mathrm{~N}$. The F502N image is dominated by the strong [O III] $\lambda 5007$ line, thus continuum contamination is negligible.

By comparing the ratio of $[\mathrm{O} \mathrm{III}] \lambda 5007 \& \lambda 4363$ emission in each pixel, a temperature map was produced, shown in Figure 1 as a contour map with contours at $500 \mathrm{~K}$ intervals between 7000 and $8500 \mathrm{~K}$, overlaid on an HST F502N image. The temperature averages about $8000 \mathrm{~K}$, and varies little across most of the surface of the nebula. This value agrees well with the results from our long-slit scanning spectra discussed below. 


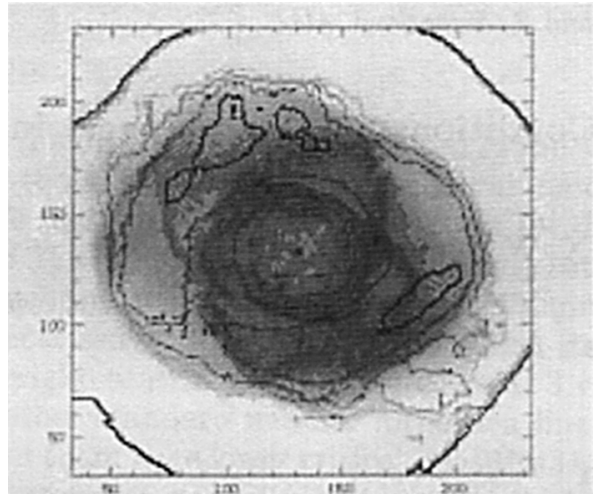

Figure 1. [O III] temperature contours overlaid on $[\mathrm{O} \mathrm{III}] \lambda 5007$ image

Hyung et al. (2001) have also produced a temperature map of NGC 6543 from the same archival HST images. They derive a higher temperature of $9000 \mathrm{~K}$ for the core of the nebula, and find that the temperature rises considerably at the outer edge of the core. We find that the temperature remains reasonably constant up to $15^{\prime \prime}$ away from the central star. Beyond this we do not believe the signal to noise ratio of the $\mathrm{F} 437 \mathrm{~N}$ image is high enough to derive an accurate temperature. These differing results may be due to different treatments of the F437N image. Hyung et al. did not correct their [O III] $\lambda 4363 \mathrm{flux}$ for $\mathrm{H} \gamma$ or $\mathrm{He} \mathrm{I}$ $\lambda 4388$ emission. We find that these corrections are not negligible, amounting to $30 \%$ of the total correction (which accounts for $53 \%$ of the flux in $\mathrm{F} 437 \mathrm{~N}$ ). Also, their continuum correction was derived using Echelle spectra of two small regions of NGC 6543, while ours is based on a spectrum of the entire nebula.

Optical, IUE and ISO spectra of NGC 6543 were analysed. The optical spectra were obtained at the WHT $4.2 \mathrm{~m}$ telescope with the ISIS double spectrograph, by uniformly scanning a long-slit across the nebula, thus yielding average spectra for the whole nebula. The Balmer jump and [O III] temperatures agree very well, at $8340 \mathrm{~K}$ and $7940 \mathrm{~K}$ respectively. From the standard diagnostics a temperature of $8000 \mathrm{~K}$ and a density of $5000 \mathrm{~cm}^{-3}$ were adopted. Abundances were calculated from both ORLs and CELs: the results are shown in Table 1.

Table 1. Ionic Abundances in units such that $\log N(\mathrm{H})=12.0$

\begin{tabular}{lcccccccc} 
& $\mathrm{He}$ & $\mathrm{C}$ & $\mathrm{N}$ & $\mathrm{O}$ & $\mathrm{Ne}$ & $\mathrm{S}$ & $\mathrm{Ar}$ & $\mathrm{Cl}$ \\
\hline ORLs & 11.07 & 8.89 & 8.77 & 9.12 & 8.74 & & & \\
CELs & & 8.46 & 8.60 & 8.91 & 8.47 & 7.15 & 6.50 & 5.41 \\
\hline
\end{tabular}

\section{References}

Benjamin, R.A., Skillman, D.E., Smits, D.P. 1999, ApJ, 514, 307

Brown, R. L., \& Mathews, W. G. 1970, ApJ, 160, 939

Hyung, S., Mellema, G., Lee, S.-J., Kim, H. 2001, A\&A, 378, 587

Storey, P.J., \& Hummer, D.G. 1995, MNRAS, 272, 41 